\title{
Clinical Characteristics of an Internet-Based Cohort of Patient-Reported Diagnosis of Granulomatosis With Polyangiitis and Microscopic Polyangiitis: Observational Study
}

Jason Michael Springer $^{1^{*}}$, MD, MS; Tanaz A Kermani ${ }^{2 *}$, MD, MS; Antoine Sreih ${ }^{3}$, MD; Dianne G Shaw ${ }^{4}$, MA; Kalen Young $^{4}$, MA; Cristina M Burroughs ${ }^{5}$, BS; Peter A Merkel ${ }^{3,6}$, MD, MPH

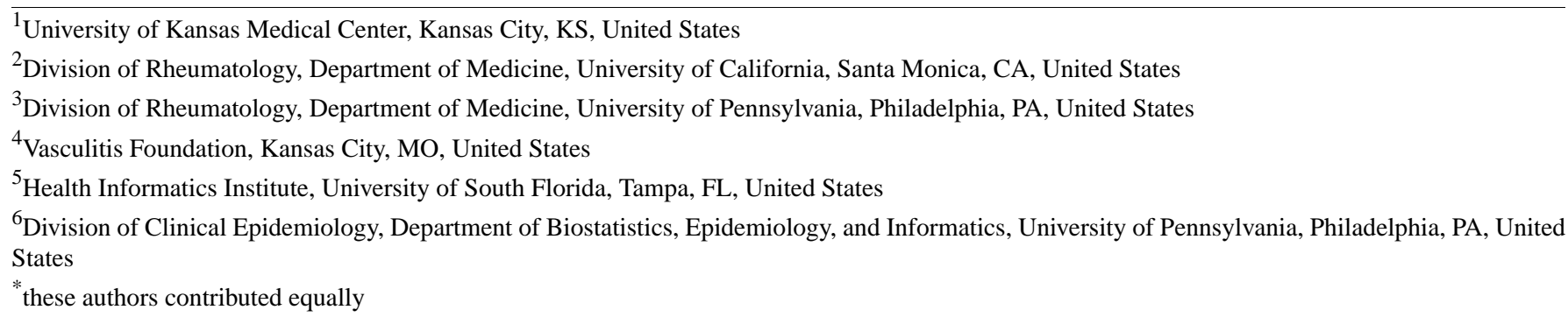

\section{Corresponding Author:}

Jason Michael Springer, MD, MS

University of Kansas Medical Center

3901 Rainbow Blvd MS 2026

Kansas City, KS, 66160

United States

Phone: 19135886009

Email: jspringer2@kumc.edu

\section{Abstract}

Background: Utilizing the traditional centers of excellence approach to conduct clinical trials involving rare diseases remains challenging. Patient-based registries have been shown to be both feasible and valid in several other diseases.

Objective: This report outlines the clinical characteristics of a large internet registry cohort of participants with a self-reported diagnosis of granulomatosis with polyangiitis or microscopic polyangiitis.

Methods: Patients with a self-reported diagnosis of granulomatosis with polyangiitis or microscopic polyangiitis in an internet-based prospective longitudinal cohort (from the Vasculitis Patient-Powered Research Network) were included. Data on symptoms, diagnostic testing, and treatment were collected using standardized questionnaires.

Results: The study compared patients with granulomatosis with polyangiitis $(n=762)$ and patients with microscopic polyangiitis $(n=164)$. Of the cohort, $97.7 \%(904 / 925)$ reported the diagnosis had been confirmed by a physician. Compared to microscopic polyangiitis, patients with granulomatosis with polyangiitis reported significantly more ear, nose, and throat manifestations (granulomatosis with polyangiitis: 641/723, 88.7\%; microscopic polyangiitis: 89/164, 54.3\%; $z=10.42, P<.001$ ), fevers (granulomatosis with polyangiitis: $325 / 588,55.3 \%$; microscopic polyangiitis: $64 / 139,46.0 \%$; $z=1.96, P=.05$ ), joint involvement (granulomatosis with polyangiitis: $549 / 688,79.8 \%$; microscopic polyangiitis: $106 / 154,68.8 \%$; $z=2.96, P=.003$ ), and pulmonary involvement (granulomatosis with polyangiitis: 523/734, 71.3\%; microscopic polyangiitis: $90 / 154,58.4 \% ; z=3.13, P=.002$ ). Compared to microscopic polyangiitis, patients with granulomatosis with polyangiitis reported significantly less renal involvement (granulomatosis with polyangiitis: 457/743, 61.5\%; microscopic polyangiitis: $135 / 163,82.8 \%$; $z=-5.18, P<.001$ ) and renal transplantation (granulomatosis with polyangiitis: 10/721, 1.4\%; microscopic polyangiitis: $7 / 164,4.3 \% ; z=-2.43, P=.02$ ). Antineutrophil cytoplasmic antibody positivity was reported in $94.2 \%(652 / 692)$ of patients with granulomatosis with polyangiitis and $96.1 \%$ (147/153) of patients with microscopic polyangiitis. A biopsy showing vasculitis was reported in $77.0 \%$ (562/730) of patients with granulomatosis with polyangiitis and $81.9 \%$ (131/160) of patients with microscopic polyangiitis.

Conclusions: In this large, internet-based cohort of patients with a self-reported diagnosis of granulomatosis with polyangiitis or microscopic polyangiitis, disease manifestations were consistent with expectations for each type of vasculitis. Given the rarity 
of these and other vasculitides, conducting some types of research through internet-based registries may provide an efficient alternative to inperson, center-of-excellence clinical trials.

(J Med Internet Res 2020;22(7):e17231) doi: 10.2196/17231

\section{KEYWORDS}

granulomatosis with polyangiitis; microscopic polyangiitis; vasculitis; patient-reported outcomes; patient registry; electronic health records; questionnaire; online cohort

\section{Introduction}

Granulomatosis with polyangiitis and microscopic polyangiitis are forms of antineutrophil cytoplasmic antibody-associated vasculitis that primarily target small arteries. These are rare diseases, with annual prevalence of granulomatosis with polyangiitis estimated from 24 to 160 per $1,000,000$ and annual prevalence of microscopic polyangiitis estimated from 39 to 94 per 1,000,000 [1]. There are several challenges in conducting clinical trials involving rare diseases, including the need to involve multiple centers, high costs, and other logistical challenges. Novel methods for obtaining both meaningful and reliable data are needed. Multiple studies [2-6] of other diseases have proven the validity of patient-reported diagnoses and outcomes. The aims of this study were to describe the self-reported clinical features of patients with granulomatosis with polyangiitis and microscopic polyangiitis who participated in the Vasculitis Patient-Powered Research Network and to establish to what extent this internet-based cohort is representative of the general population of patients with these forms of antineutrophil cytoplasmic antibody-associated vasculitis.

\section{Methods}

Established in 2014, the Vasculitis Patient-Powered Research Network is an international, internet-based prospective longitudinal registry of patient- or caregiver-reported information. The Vasculitis Patient-Powered Research Network was established as a partnership between the Vasculitis Clinical Research Network (a vasculitis research network) and the Vasculitis Foundation (the largest patient advocacy group for vasculitis). The network represents a collaboration among a variety of vasculitis stakeholders including patients, patient advocacy organizations, academic clinical investigators, expert clinicians, biomedical informaticians, methodologists, and funding organizations. Patient-partners are an integral part of team and are involved in strategically planning, developing, reviewing, and approving research studies. Patient-partners receive training in patient participation in research.

The Vasculitis Patient-Powered Research Network is the largest patient-based registry for primary systemic vasculitis with over 3000 patients enrolled to date; patients (or caregivers) consent to participate in studies and provide self-reported information longitudinally using the internet-based platform [7]. For this study, advertisement for recruitment was done via social media, the Vasculitis Foundation website, and flyers at national and regional vasculitis conferences.
Only patients with a self-reported diagnosis of granulomatosis with polyangiitis and microscopic polyangiitis were included in this study. Patients provided consent online and were enrolled between November 2014 and May 2019. The data were obtained via convenience (or opportunity) sampling methods. Using the internet-based patient portal, participants filled out standardized questionnaires (open survey, Multimedia Appendix 1) in English which requested information such as demographics (age, gender, ethnicity), signs and symptoms of vasculitis at the time of diagnosis, results of diagnostic studies performed (ie, laboratory testing, biopsies, imaging, etc), prescribed treatments, and outcomes. For each item, respondents could select yes, no, or $I$ don't know and were able to review or change previously answered questions. Responses that were left blank or where the answer was not known were excluded from the analysis. Patient participation was encouraged by sending email reminders to participants; however, no incentives were offered for completion of the questionnaires. Multiple entries by the same individual were prevented through the use of password-protected user log-ins.

This study was approved by the institutional review board of the University of South Florida. To address data completeness and compliance, the Vasculitis Patient-Powered Research Network operates a comprehensive data compliance strategy using a variety of tools and approaches. The data compliance reports are regularly monitored by the network and data managers of the Vasculitis Patient-Powered Research Network to identify emerging trends. If a participant has not completed all forms, a series of automated email reminders to the participant are triggered. After an initial, generic reminder email message has been sent, a form-specific email reminder is sent. This message specifies which forms are incomplete and also describes the scientific significance and need for the information requested by each form.

Data were analyzed to compare clinical manifestations and diagnostic testing of patients with granulomatosis with polyangiitis to those of patients with microscopic polyangiitis. Two-tailed $z$ scores were performed for comparisons of proportions. Two-tailed independent $t$ tests were used for comparisons of means and medians. $P$ values $\leq .05$ were considered significant.

\section{Results}

\section{Participant Characteristics}

\section{Granulomatosis With Polyangiitis}

A total of 762 participants reported a diagnosis of granulomatosis with polyangiitis; $518(68.0 \%)$ were female and 
$244(32.0 \%)$ were male. The median age of patients at the onset of symptoms was 45 (IQR 31-57) for 619 respondents, and the median age at diagnosis was 48 (IQR 35-57) for 683 respondents. Out of 761 respondents, $248(32.6 \%)$ reported their disease as active, $465(61.1 \%)$ reported their disease as being in remission, and $48(6.3 \%)$ reported they were unsure. Respondents $(648 / 727,89.1 \%)$ reported seeing one or more of the following specialists: rheumatologist $(528 / 727,72.6 \%)$, nephrologist $(202 / 727,27.8 \%)$, pulmonologist $(139 / 727,19.1 \%)$, otolaryngologist (135/727, 18.6\%), neurologist (29/727, 4.0\%),

Figure 1. Heat maps showing geographic distribution of participants with granulomatosis with polyangiitis and microscopic polyangiitis internationally. immunologist $(9 / 727,1.2 \%)$, dermatologist $(3 / 727,0.4 \%)$. Respondents $(720 / 762,94.5 \%)$ reported their country of origin as the United States $(572 / 720,79.4 \%$, of which state unreported: 72/572 and state reported: 500/572; northeastern states: 102/500, 20.4\%; midwestern states: 132/500, 26.4\%; southern states: 164/500, 32.8\%; western states: 102/500, 20.4\%), Canada (62/720, 8.6\%), United Kingdom (36/720, 5.0\%), Australia $(18 / 720,2.5 \%)$, or other $(32 / 720,4.4 \%)$ (Figure 1 and Figure 2).

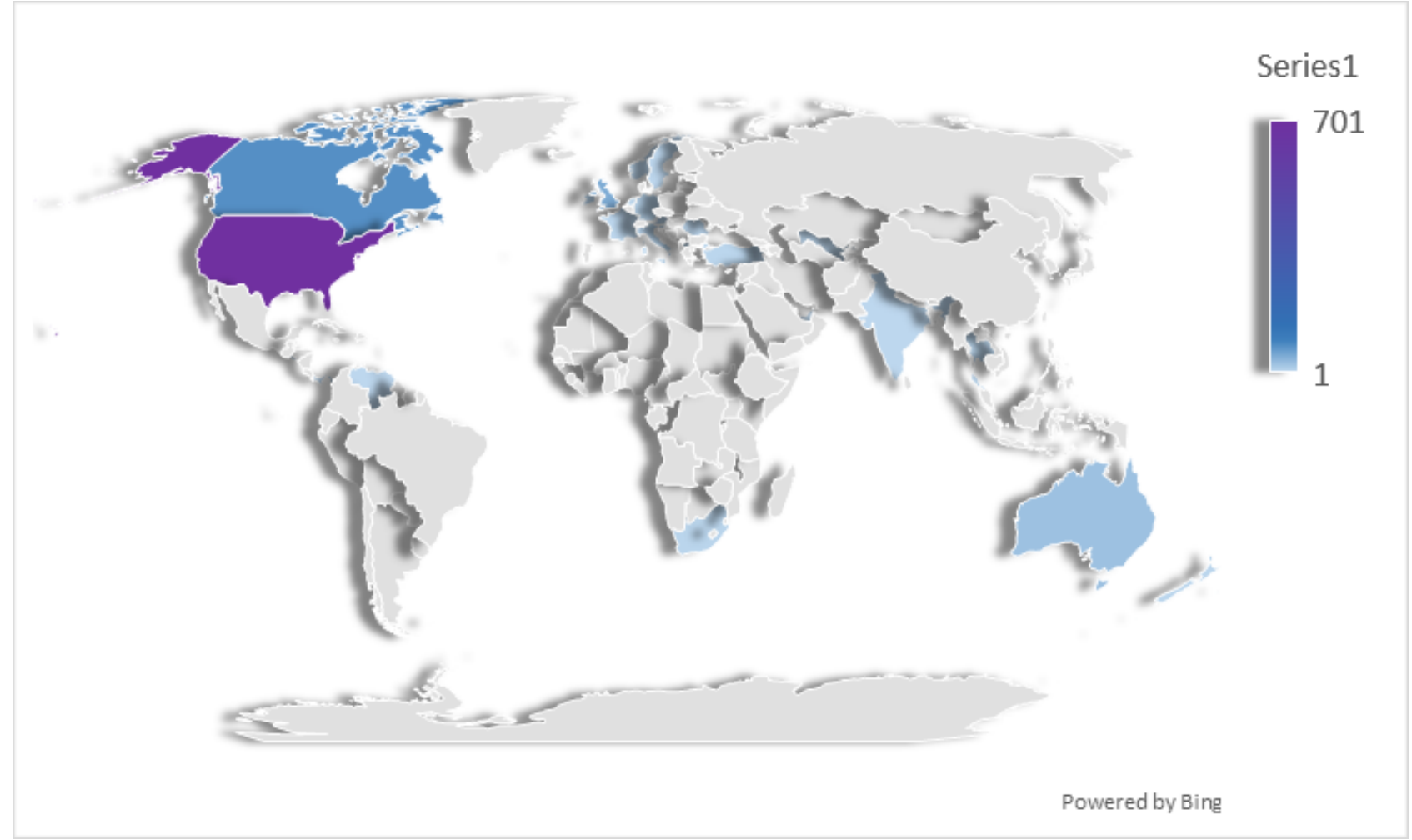


Figure 2. Heat maps showing geographic distribution of participants with granulomatosis with polyangiitis and microscopic polyangiitis within the United States of America.

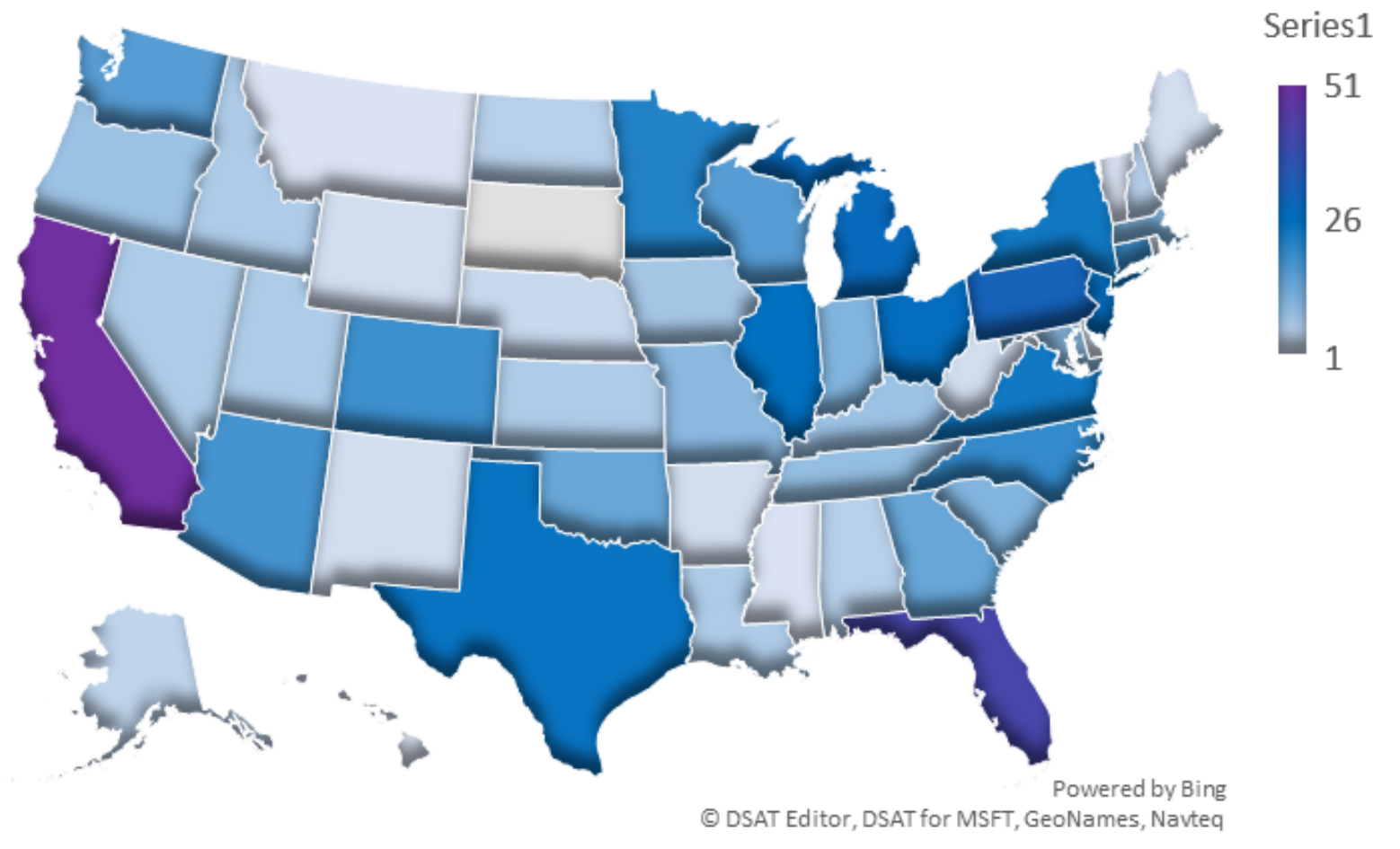

\section{Microscopic Polyangiitis}

A total of 164 participants reported a diagnosis of microscopic polyangiitis; $133(81.1 \%)$ were female and 31 (18.9\%) were male. The median age of patients at the onset of symptoms was 52 (IQR 36-61) for 129 respondents, and the median age at diagnosis was 53 (IQR 41-62) for 158 respondents. Out of 160 respondents, 55 (34.4\%) reported their disease as active, 96 $(60.0 \%)$ reported their disease as being in remission, and 13 $(8.1 \%)$ reported they were unsure. Respondents (141/160, $88.1 \%$ ) reported seeing one or more of the following specialists: rheumatologist $(95 / 160,59.4 \%)$, nephrologist $(85 / 160,53.1 \%)$, pulmonologist $(28 / 160,17.5 \%)$, neurologist $(6 / 160,3.8 \%)$, immunologist $(3 / 160,1.9 \%)$, dermatologist $(2 / 160,1.2 \%)$ and otolaryngologist $(1 / 160,0.6 \%)$. Respondents $(150 / 164,91.5 \%)$ reported their country of origin as the United States (121/150, $80.6 \%$, of which state unreported: $12 / 121$ and state reported: 109/121; northeastern states: $15 / 109,13.8 \%$; midwestern states: 30/109, 27.6\%; southern states: 31/109, 28.4\%; western states: 33/109, 30.3\%), Canada (10/150, 6.6\%), United Kingdom $(8 / 150,5.3 \%)$, Australia $(5 / 150,3.3 \%)$, or other $(6 / 150,4.0 \%)$

\section{Self-Reported Manifestations}

In patients with granulomatosis with polyangiitis, the most common self-reported manifestations were nasal or sinus in $82.9 \%(600 / 723)$, joint pain in $79.8 \%(549 / 688)$, pulmonary in $71.3 \%(523 / 734)$, peripheral nerve in $62.8 \%(411 / 654)$, and renal in $61.5 \%(457 / 743)$ of respondents. Pulmonary-renal syndrome (diffuse alveolar hemorrhage in combination with renal disease) was reported by $26.9 \%$ (184/684) of participants. Venous thromboembolisms were reported by $13.7 \%(96 / 701)$ of respondents.
In patients with microscopic polyangiitis, the most common self-reported manifestations were renal in $82.8 \%(135 / 163)$, joint pain in $68.8 \%(106 / 154)$, peripheral nerve in $64.6 \%$ $(95 / 147)$, nasal or sinus in $61.0 \%(89 / 146)$, rash in $59.6 \%$ $(93 / 156)$, and pulmonary in $58 \%(90 / 154)$ of respondents. Pulmonary-renal syndrome was reported by $29 \%$ (47/161). Venous thromboembolism was reported in $13.6 \%$ (21/154) of respondents. Patients also reported isolated renal manifestations without other organ manifestations $(14 / 164,8.5 \%)$, although constitutional symptoms such as myalgia (1/164, 0.6\%), fever $(2 / 164,1.2 \%)$, and weight loss $(6 / 164,3.7 \%)$ were reported.

\section{Diagnostic Testing}

Confirmation of diagnosis by a physician was reported by $97.5 \%$ (742/761) of respondents with granulomatosis with polyangiitis. Patients with granulomatosis with polyangiitis underwent antineutrophil cytoplasmic antibody testing $(652 / 692,94.2 \%)$, laboratory testing $(566 / 762,74.3 \%)$, biopsy $(475 / 762,62.3 \%)$, and imaging (292/762, 38.3\%) for diagnosis; symptom-based diagnosis was also reported $(533 / 762,69.9 \%)$. Out of 730 respondents, $562(77.0 \%)$ reported having a biopsy showing vasculitis at some point, of which the biopsy sites included kidney $(283 / 562,50.4 \%)$, lung $(192 / 562,34.2 \%)$, nasal or sinus $(146 / 562,26.0 \%)$, skin $(70 / 562,12.5 \%)$, nerve $(10 / 562,1.8 \%)$, artery $(7 / 562,1.2 \%)$, and other sites $(36 / 562,6.4 \%)$.

Confirmation of diagnosis by a physician was reported by $98.8 \%$ $(162 / 164)$ of respondents with microscopic polyangiitis. Patients with microscopic polyangiitis underwent antineutrophil cytoplasmic antibody testing $(147 / 153,96.1 \%)$, laboratory testing $(129 / 164,78.7 \%)$, biopsy $(126 / 164,76.8 \%)$, and imaging $(69 / 164,42.1 \%)$ for diagnosis; symptom-based diagnosis was also reported $(110 / 164,67.1 \%)$. Of 160 respondents, 131 
$(81.9 \%)$ reported having a biopsy showing vasculitis at some point, of which the biopsy sites included kidney (103/160, $78.6 \%)$, lung $(20 / 160,15.3 \%)$, skin $(15 / 160,11.5 \%)$, nerve $(6 / 160,4.6 \%)$, nasal or sinus $(4 / 160,3.1 \%)$, artery $(3 / 160,2.3 \%)$, and other sites $(2 / 160,1.5 \%)$. Additional diagnostic testing information can be found in Multimedia Appendix 2.

\section{Medications}

For respondents with granulomatosis, the most commonly used medications included glucocorticoids (707/762, 92.8\%), cyclophosphamide (total: 439/762, 57.6\%; oral: 313/762, 41.1\%; intravenous: 226/762, 29.7\%), sulfamethoxazole and trimethoprim $(418 / 762,54.9 \%)$, rituximab $(396 / 762,52.0 \%)$, methotrexate $(354 / 762,46.5 \%)$, azathioprine $(311 / 762,40.8 \%)$, mycophenolate $(140 / 762,18.4 \%)$, and intravenous immunoglobulin (38/762, 5.0\%); 8.4\% (64/762) reported receiving plasma exchange at some point. For respondents with microscopic granulomatosis, the most commonly used medications included glucocorticoids (151/161, 93.8\%), rituximab (89/161, 55.3\%), cyclophosphamide (total: 79/161, 49.1\%; oral: 44/161, 27.3\%; intravenous: 49/161, 30.4\%), azathioprine in $(79 / 161,49.1 \%)$, sulfamethoxazole and trimethoprim $(53 / 161,32.9 \%)$, mycophenolate $(39 / 161,24.2 \%)$, methotrexate $(34 / 161,21.1 \%)$, and intravenous immunoglobulin (9/161, 5.6\%); 9.9\% (16/161) reported receiving plasma exchange at some point. Additional data on the medications used by patients with granulomatosis with polyangiitis and by patients with microscopic polyangiitis to treat their vasculitis can be found in Multimedia Appendix 3.

\section{Granulomatosis With Polyangiitis Versus Microscopic Polyangiitis}

Compared to those with a self-reported diagnosis of microscopic polyangiitis, those with a self-reported diagnosis of granulomatosis with polyangiitis were younger at both onset of symptoms ( 45 years of age versus 52 years of age, $\mathrm{t}_{746}=-2.46$,
$P=.01$ ) and diagnosis (48 years of age versus 53 years of age, $\left.\mathrm{t}_{839}=-2.60, \quad P=.01\right)$. Patients with granulomatosis with polyangiitis reported more sinonasal disease $(83.0 \%$ versus $61.0 \%, z=5.99, P<.001)$, hearing loss ( $48.3 \%$ versus $15.3 \%$, $z=7.28, P<.001)$, tracheal involvement $(30.0 \%$ versus $9.8 \%$, $z=4.80, P<.001)$, pulmonary involvement $(71.3 \%$ versus $58.4 \%$, $z=3.13, P=.002)$, eye involvement $(54.2 \%$ versus $33.8 \%, z=3.14$, $P=.002)$, joint involvement $(79.8 \%$ versus $68.8 \%, z=2.96$, $P=.003)$ and fevers $(55.3 \%$ versus $46.0 \%, z=1.96, P=.050)$ than patients with microscopic polyangiitis; whereas, those with microscopic polyangiitis reported more renal involvement $(82.8 \%$ versus $61.5 \%, z=-5.18, P<.001)$ and were more likely to have undergone a renal transplant $(4.3 \%$ versus $1.4 \%$, $z=-2.43, P=.02)$ than patients with granulomatosis with polyangiitis (Table 1). There was no difference in the proportion of venous thromboembolism $(13.7 \%$ versus $13.6 \%, z=0.02$, $P=.99$ ), no difference in the percentage of patients reporting a positive antineutrophil cytoplasmic antibody test $(94.2 \%$ versus $96.1 \%, z=-0.92, P=.36$ ), and no difference in the percentage of patients reporting a diagnosis based on biopsy $(77.0 \%$ versus $81.9 \%, z=-1.35, P=.18)$. There were more diagnoses from lung (34.2\% versus $15.3 \%, z=4.23, P<.001)$ and nasal or sinus biopsies $(26.0 \%$ versus $3.1 \%, z=5.74, P<.001)$ in participants with granulomatosis with polyangiitis than those in participants with microscopic polyangiitis. Conversely, there were more diagnoses from kidney biopsy in participants with microscopic polyangiitis $(78.6 \%$ versus $50.4 \%, z=-5.87, P<.001)$ than those in participants with granulomatosis with polyangiitis. A biopsy was more likely to have been performed in participants with microscopic polyangiitis than in those with granulomatosis with polyangiitis $(76.8 \%$ versus $62.3 \%, z=-3.53, P<.001)$. Oral cyclophosphamide $(z=3.25, P=.001)$, methotrexate $(z=5.92$, $P<.001)$, and sulfamethoxazole and trimethoprim $(z=5.06$, $P<.001)$ were more commonly used by patients with granulomatosis with polyangiitis than by those with microscopic polyangiitis (Multimedia Appendix 3). 
Table 1. Self-reported clinical manifestations.

\begin{tabular}{|c|c|c|c|c|c|c|}
\hline \multirow[t]{2}{*}{ Clinical Manifestation } & \multicolumn{2}{|c|}{ Granulomatosis with polyangiitis } & \multicolumn{2}{|c|}{ Microscopic polyangiitis } & \multirow[b]{2}{*}{$Z$ score } & \multirow[b]{2}{*}{$P$ value } \\
\hline & $\mathrm{N}^{\mathrm{a}}$ & $\mathrm{n}(\%)$ & $\mathrm{N}^{\mathrm{a}}$ & $\mathrm{n}(\%)$ & & \\
\hline Rash & 698 & $401(57.4)$ & 156 & $93(59.6)$ & -0.50 & .62 \\
\hline Weight loss & 690 & $400(58.0)$ & 151 & $86(57.0)$ & 0.23 & .82 \\
\hline Fever & 588 & $325(55.3)$ & 139 & $64(46.0)$ & 1.96 & .05 \\
\hline Joint & 688 & $549(79.8)$ & 154 & $106(68.8)$ & 2.96 & .003 \\
\hline \multicolumn{7}{|l|}{ Ear, nose, and throat } \\
\hline Any & 723 & $641(88.7)$ & 164 & $89(54.3)$ & 10.42 & $<.001$ \\
\hline Nasal/sinus symptoms & 723 & $600(83.0)$ & 146 & $89(61.0)$ & 5.99 & $<.001$ \\
\hline Hearing loss & 666 & $322(48.3)$ & 144 & $22(15.3)$ & 7.28 & $<.001$ \\
\hline Tracheal & 610 & $183(30.0)$ & 133 & $13(9.8)$ & 4.80 & $<.001$ \\
\hline \multicolumn{7}{|l|}{ Pulmonary } \\
\hline Any & 734 & $523(71.3)$ & 154 & $90(58.4)$ & 3.13 & .002 \\
\hline Alveolar hemorrhage & 700 & $282(40.3)$ & 161 & $58(36.0)$ & 1.00 & .32 \\
\hline \multicolumn{7}{|l|}{ Renal } \\
\hline Any & 743 & $457(61.5)$ & 163 & $135(82.8)$ & -5.18 & $<.001$ \\
\hline Dialysis & 721 & $73(10.1)$ & 163 & $18(11.0)$ & -0.35 & .73 \\
\hline Renal transplant & 721 & $10(1.4)$ & 164 & $7(4.3)$ & -2.43 & .02 \\
\hline Pulmonary-renal & 684 & $184(26.9)$ & 161 & $47(29.2)$ & -0.59 & .56 \\
\hline Peripheral nerve & 654 & $411(62.8)$ & 147 & $95(64.6)$ & -0.40 & .69 \\
\hline Gastrointestinal & 683 & $14(2.0)$ & 147 & $4(2.7)$ & -0.51 & .61 \\
\hline Venous thromboembolism & 701 & $96(13.7)$ & 154 & $21(13.6)$ & 0.02 & .99 \\
\hline Pericardial & 609 & $49(8.0)$ & 132 & $6(4.6)$ & 1.39 & .16 \\
\hline Eye & 609 & $330(54.2)$ & 151 & $51(33.8)$ & 3.14 & .002 \\
\hline
\end{tabular}

${ }^{\mathrm{a}}$ The number of patients who responded yes or no (unknown and blank responses were excluded).

\section{Discussion}

\section{Principal Findings}

This study was conducted using exclusively patient-reported information. It is important to understand how patient-derived data may differ from those obtained through traditional physician reports used in observational cohorts and clinical trials. This is the first large, prospective cohort of patients with a self-reported diagnosis of granulomatosis with polyangiitis or microscopic polyangiitis that included detailed information from standardized forms to evaluate clinical manifestations, the results of diagnostic testing, and types of treatment. The participants were from across the United States, Canada, and from multiple other countries. An analysis [8] of this cohort found that more than $90 \%$ of patients met the 1990 American College of Rheumatology classification criteria for granulomatosis with polyangiitis and the Chapel Hill Consensus Conference definition of microscopic polyangiitis.

The type and distribution of clinical manifestations among patients in this cohort were similar to those reported for observational cohorts and in clinical trials [9-11]. Ear, nose, and throat; pulmonary; and renal manifestations were common in granulomatosis with polyangiitis, as expected, while renal and lung involvement were common in microscopic polyangiitis. The proportions of positive antineutrophil cytoplasmic antibody tests $(94.2 \%$ and $96.1 \%)$ were similar to what would be expected and to what has been in the literature [12]. It has been recognized that there is a high risk of venous thromboembolism in antineutrophil cytoplasmic antibody-associated vasculitis, especially during active disease. There was a $14 \%$ prevalence of venous thromboembolism in the combined cohort which was similar to that reported in the literature $[13,14]$.

The most common means of diagnosis reported by participants (symptom-based, laboratory testing, biopsy, and imaging) closely reflected what was emphasized by the 1990 American College of Rheumatology classification criteria for granulomatosis with polyangiitis and the Chapel Hill Consensus Conference definition of microscopic polyangiitis. Only $1 \%$ of participants were not sure of the means of their diagnosis, reflecting good insight and recall of participants regarding the basis for their original diagnosis.

There were also important differences between this cohort and center-based cohorts. First, there was a higher than expected prevalence of skin $(57.4 \%$ and $59.6 \%)$ and peripheral nerve 
involvement $(62.8 \%$ and $64.6 \%)$. This could reflect the nonspecificity of the queries where patients were asked to attribute manifestations to vasculitis. Patients may have had difficulty adjudicating whether their symptoms were caused by vasculitis or as a result of complications of therapy.

Ear, nose, and throat manifestations are a hallmark manifestation for granulomatosis with polyangiitis distinguishing it from microscopic polyangiitis. In a study [15] comparing physician-reported clinical characteristics of granulomatosis with polyangiitis and microscopic polyangiitis in observational cohorts versus randomized controlled trials, $19 \%$ patients with microscopic polyangiitis had ear, nose, and throat manifestations compared to $76 \%$ patients with granulomatosis with polyangiitis. In this study cohort, there was a statistically higher $(z=10.42$, $P<.001)$ prevalence of these manifestations in granulomatosis with polyangiitis as expected, but there was also a higher than expected prevalence in microscopic polyangiitis with nasal-sinus manifestations being reported in $54.3 \%$ of patients. This may represent the nonspecificity of queries which included asking patients to attribute manifestations to vasculitis. Alternatively, it may in part also reflect misclassification in the medical community in which patients who test positive for myeloperoxidase antineutrophil cytoplasmic antibodies are classified as having microscopic polyangiitis; and only patients who test positive for proteinase 3 antineutrophil cytoplasmic antibodies are classified as having granulomatosis with polyangiitis.

As expected, patients with microscopic polyangiitis were significantly more likely $(z=-5.18, P<.001)$ to have had renal involvement than patients with granulomatosis with polyangiitis. In the microscopic polyangiitis group, $8.5 \%$ patients had isolated renal manifestations (renal-limited vasculitis). Overall, the proportion of renal involvement was similar to that reported in the literature [12]; however, in earlier reports, up to one-third of those with antineutrophil cytoplasmic antibody-associated vasculitis with renal involvement went on to develop end-stage renal disease [16] which was higher than what was found in this study (granulomatosis with polyangiitis: 73/457, 16.0\%; microscopic polyangiitis: $18 / 135,13.3 \%$ ). This could be related to the improvement of renal outcomes in granulomatosis with polyangiitis and microscopic polyangiitis patients over time reflecting the changes in diagnosis and management $[16,17]$. This could also reflect the fact that there was a possible bias as a result of less morbidity in participants from the online portal. Furthermore, this study enrolled patients with a clinical diagnosis of granulomatosis with polyangiitis or microscopic polyangiitis; patients who had isolated renal manifestations and part of the spectrum of antineutrophil cytoplasmic antibody-associated vasculitis could have been underrepresented, especially if they had not been clinically diagnosed with granulomatosis with polyangiitis or microscopic polyangiitis by their physicians. Patients with renal-limited disease who have end-stage renal disease may also no longer be followed or monitored for vasculitis, and therefore, may not have enrolled in the study.

\section{Strengths and Limitations}

This study had several strengths. First, the study cohort is large for such rare diseases, and is geographically diverse. The data elements of interest were selected and designed by highly experienced investigators in this field and collected using standardized forms. Patient input was obtained at each stage of the process, especially on the design of the user interface for the Vasculitis Patient-Powered Research Network website.

This study had several limitations. First, direct physician confirmation of the diagnosis was not obtained as part of this study; however, based on the patient information that was provided, more than $90 \%$ of patients with granulomatosis with polyangiitis and microscopic polyangiitis met either the 1990 American College of Rheumatology Classification Criteria for granulomatosis with polyangiitis or the Chapel Hill Consensus Conference definition for microscopic polyangiitis [8]. Almost all of the participants reported physician confirmation of the diagnosis. In addition, the manifestations described by patients were similar to those reported in the literature. Second, questions that were left blank or for which the participant answered, "I don't know" were not included in the analysis. This may represent a response bias that is common among studies using self-reported data; however, for most questions this represented a small percentage of responses. Third, bias sampling may have led to an overrepresentation of certain subgroups. For instance, there were more women than men (2:1 for granulomatosis with polyangiitis and 4:3 for microscopic polyangiitis), despite the sex ratio in the general population being close to 1 for both diseases [1]. Internet-based participation in many surveys and studies is more common among women [18-20]. Age may also have been a factor in participation in this internet-based cohort. This appears to be less of an issue in this study which includes patients from across the age spectrum, including individuals older than 70 years. Fourth, the mode of survey response (internet) may have prevented some patients who lack access or higher education and since many of the participants were recruited through the Vasculitis Foundation, and it may also represent a bias toward participants who are savvier about their disease.

\section{Conclusions}

Granulomatosis with polyangiitis and microscopic polyangiitis are both rare (also known as orphan diseases), which makes clinical research for these disorders difficult and requires the use of multiple centers which can be financially and logistically challenging. Patient registries offer an alternative to the centers of excellence approach to conducting clinical research. The data reported herein for an internet-based cohort demonstrated the feasibility of such registries across broad geographic regions and the high level of comparability between an internet-based and traditional academic center-based participant populations. These data provide investigators and patients with confidence that internet-based patient-reported data are reliable and can be used to conduct novel, cost-efficient medical research. Such internet-based registries offer an advantage in capturing participant data from those who would not otherwise be able to participate in studies. 


\section{Acknowledgments}

The Vasculitis Patient-Powered Research Network has received support from the Patient-Centered Research Institute (PCORI award PPRN-1306-04758) and through the Vasculitis Clinical Research Consortium. The Vasculitis Clinical Research Consortium is part of the Rare Diseases Clinical Research Network, an initiative of the Office of Rare Diseases Research, National Center for Advancing Translational Science. The Vasculitis Clinical Research Consortium is funded through a collaboration between National Center for Advancing Translational Science and the National Institute of Arthritis and Musculoskeletal and Skin Diseases (U54 AR057319).

\section{Conflicts of Interest}

PAM reports receiving funds for various activities in the past two years. They have done consulting for the following institutions: AbbVie, AstraZeneca, Biogen, Boeringher-Ingelheim, Bristol-Myers Squibb, Celgene, ChemoCentryx, CSL Behring, Forbius, Genentech/Roche, Genzyme/Sanofi, GlaxoSmithKline, InflaRx, Insmed, Jannsen, Kiniksa, Magenta, Pfizer, Sparrow, Talaris. They recieved research support from the following institutions: AstraZeneca, Boeringher-Ingelheim, Bristol-Myers Squibb, Celgene, ChemoCentryx, Forbius, Genentech/Roche, Genzyme/Sanofi, GlaxoSmithKline, InflaRx. They recieve royalties from the following institutions: UpToDate.

\section{Multimedia Appendix 1}

Vasculitis Patient-Powered Research Network online questionnaire forms for granulomatosis with polyangiitis and microscopic polyangiitis.

[DOCX File , 65 KB-Multimedia Appendix 1]

\section{Multimedia Appendix 2}

Patient-reported testing methods to establish a diagnosis of granulomatosis with polyangiitis or microscopic polyangiitis. [DOCX File, 15 KB-Multimedia Appendix 2]

\section{Multimedia Appendix 3}

Patient-reported medications used to treat granulomatosis with polyangiitis and microscopic polyangiitis. [DOCX File, 14 KB-Multimedia Appendix 3]

\section{References}

1. Ntatsaki E, Watts RA, Scott DGI. Epidemiology of ANCA-associated vasculitis. Rheum Dis Clin North Am 2010 Aug;36(3):447-461. [doi: 10.1016/j.rdc.2010.04.002] [Medline: 20688243]

2. Peeters GMEE, Tett SE, Dobson AJ, Mishra GD. Validity of self-reported osteoporosis in mid-age and older women. Osteoporos Int 2013 Mar;24(3):917-927. [doi: 10.1007/s00198-012-2033-7] [Medline: 22673826]

3. Randell RL, Long MD, Cook SF, Wrennall CED, Chen W, Martin CF, et al. Validation of an internet-based cohort of inflammatory bowel disease (CCFA partners). Inflamm Bowel Dis 2014 Mar;20(3):541-544 [FREE Full text] [doi: 10.1097/01.MIB.0000441348.32570.34] [Medline: 24451221]

4. Pastorino S, Richards M, Hardy R, Abington J, Wills A, Kuh D, National Survey of HealthDevelopment ScientificData Collection Teams. Validation of self-reported diagnosis of diabetes in the 1946 British birth cohort. Prim Care Diabetes 2015 Oct;9(5):397-400 [FREE Full text] [doi: 10.1016/j.pcd.2014.05.003] [Medline: 25037637]

5. Jackson CA, Mishra GD, Tooth L, Byles J, Dobson A. Moderate agreement between self-reported stroke and hospital-recorded stroke in two cohorts of Australian women: a validation study. BMC Med Res Methodol 2015 Jan 23;15:7 [FREE Full text] [doi: 10.1186/1471-2288-15-7] [Medline: 25613556]

6. Eichler GS, Cochin E, Han J, Hu S, Vaughan TE, Wicks P, et al. Exploring concordance of patient-reported information on PatientsLikeMe and medical claims data at the patient level. J Med Internet Res 2016 May 12;18(5):e110 [FREE Full text] [doi: 10.2196/jmir.5130] [Medline: 27174602]

7. Vasculitis-Patient Powered Research Network website. URL: https://www.vpprn.org/ [accessed 2020-03-30]

8. Kermani TS, Sreih A, Shaw D, Young K, Burroughs C, Merkel P. Arthritis Rheum. In: Accuracy of self-reported diagnosis of antineutrophil cytoplasmic antibody-associated vasculitis abstract. 2019 Presented at: American College of Rheumatology Annual Meeting; November 8-13 2019; Atlanta URL: https://acrabstracts.org/abstract/ accuracy-of-self-reported-diagnosis-of-antineutrophil-cytoplasmic-antibody-associated-vasculitis/

9. Stone JH, Merkel PA, Spiera R, Seo P, Langford CA, Hoffman GS, RAVE-ITN Research Group. Rituximab versus cyclophosphamide for ANCA-associated vasculitis. N Engl J Med 2010 Jul 15;363(3):221-232 [FREE Full text] [doi: 10.1056/NEJMoa0909905] [Medline: 20647199] 
10. Pagnoux C, Mahr A, Hamidou MA, Boffa J, Ruivard M, Ducroix J, French Vasculitis Study Group. Azathioprine or methotrexate maintenance for ANCA-associated vasculitis. N Engl J Med 2008 Dec 25;359(26):2790-2803. [doi: 10.1056/NEJMoa0802311] [Medline: 19109574]

11. Guillevin L, Pagnoux C, Karras A, Khouatra C, Aumaître O, Cohen P, French Vasculitis Study Group. Rituximab versus azathioprine for maintenance in ANCA-associated vasculitis. N Engl J Med 2014 Nov 06;371(19):1771-1780. [doi: 10.1056/NEJMoa1404231] [Medline: 25372085]

12. Solans-Laqué R, Fraile G, Rodriguez-Carballeira M, Caminal L, Castillo MJ, Martínez-Valle F, Spanish Registry of systemic vasculitis (REVAS) from the Autoimmune Diseases Study Group (GEAS) of the Spanish Society of Internal Medicine (SEMI). Clinical characteristics and outcome of Spanish patients with ANCA-associated vasculitides: impact of the vasculitis type, ANCA specificity, and treatment on mortality and morbidity. Medicine (Baltimore) 2017 Feb;96(8):e6083 [FREE Full text] [doi: 10.1097/MD.0000000000006083] [Medline: 28225490]

13. Merkel PA, Lo GH, Holbrook JT, Tibbs AK, Allen NB, Davis JC, Wegener's Granulomatosis Etanercept Trial Research Group. Brief communication: high incidence of venous thrombotic events among patients with Wegener granulomatosis: the Wegener's Clinical Occurrence of Thrombosis (WeCLOT) Study. Ann Intern Med 2005 Apr 19;142(8):620-626. [doi: 10.7326/0003-4819-142-8-200505030-00011] [Medline: 15838068]

14. Allenbach Y, Seror R, Pagnoux C, Teixeira L, Guilpain P, Guillevin L, French Vasculitis Study Group. High frequency of venous thromboembolic events in Churg-Strauss syndrome, Wegener's granulomatosis and microscopic polyangiitis but not polyarteritis nodosa: a systematic retrospective study on 1130 patients. Ann Rheum Dis 2009 Apr;68(4):564-567. [doi: 10.1136/ard.2008.099051] [Medline: 19015208]

15. Pagnoux C, Carette S, Khalidi NA, Walsh M, Hiemstra TF, Cuthbertson D, french Vasculitis Study Group (FVSG), European Vasculitis Society (EUVAS)Vasculitis Clinical Research Consortium (VCRC). Comparability of patients with

ANCA-associated vasculitis enrolled in clinical trials or in observational cohorts. Clin Exp Rheumatol 2015;33(2 Suppl 89):S-77 [FREE Full text] [Medline: 26016754]

16. Rhee RL, Hogan SL, Poulton CJ, McGregor JAG, Landis JR, Falk RJ, et al. Trends in long-term outcomes among patients with antineutrophil cytoplasmic antibody-associated vasculitis with renal disease. Arthritis Rheumatol 2016 Jul;68(7):1711-1720 [FREE Full text] [doi: 10.1002/art.39614] [Medline: 26814428]

17. Whatmough S, Fernandez S, Sweeney N, Howell L, Dhaygude A. Comparing outcomes of biopsy-proven anti-neutrophil cytoplasmic autoantibody-associated glomerulonephritis patients treated with cyclophosphamide in the 20th and 21st centuries: a 23-year study. Clin Kidney J 2019 Feb;12(1):42-48 [FREE Full text] [doi: 10.1093/ckj/sfy084] [Medline: 30746129]

18. Curtin R, Presser S, Singer E. The effects of response rate changes on the index of consumer sentiment. Public Opin Q 2000;64(4):413-428. [doi: 10.1086/318638] [Medline: 11171024$]$

19. Singer E, Van Hoewyk J, Maher MP. Experiments with incentives in telephone surveys. Public Opin Q 2000;64(2):171-188. [doi: 10.1086/317761] [Medline: 10984332]

20. Abdou NI, Kullman GJ, Hoffman GS, Sharp GC, Specks U, McDonald T, et al. Wegener's granulomatosis: survey of 701 patients in North America. Changes in outcome in the 1990s. J Rheumatol 2002 Feb;29(2):309-316. [Medline: 11838848]

Edited by G Eysenbach; submitted 24.02.20; peer-reviewed by R Richesson, D Jayne; comments to author 01.04.20; revised version
received 30.04.20; accepted 14.05.20; published 20.07 .20
Please cite as:
Springer JM, Kermani TA, Sreih A, Shaw DG, Young K, Burroughs CM, Merkel PA
Clinical Characteristics of an Internet-Based Cohort of Patient-Reported Diagnosis of Granulomatosis With Polyangiitis and
Microscopic Polyangiitis: Observational Study
J Med Internet Res 2020;22(7):e17231
URL: $\underline{\text { htps://www.jmir.org/2020/7/e17231 }}$
doi: $\underline{10.2196 / 17231}$
PMID: $\underline{32459634}$

(CJason Michael Springer, Tanaz A Kermani, Antoine Sreih, Dianne G Shaw, Kalen Young, Cristina M Burroughs, Peter A Merkel. Originally published in the Journal of Medical Internet Research (http://www.jmir.org), 20.07.2020. This is an open-access article distributed under the terms of the Creative Commons Attribution License (https://creativecommons.org/licenses/by/4.0/), which permits unrestricted use, distribution, and reproduction in any medium, provided the original work, first published in the Journal of Medical Internet Research, is properly cited. The complete bibliographic information, a link to the original publication on http://www.jmir.org/, as well as this copyright and license information must be included. 